\title{
INTRINSICALLY ERGODIC SYSTEMS
}

BY B. WEISS

Communicated by Henry McKean, Jr., April 9, 1970

Let $\phi$ be a continuous mapping of a compact metric space $X$ into itself and $M_{\phi}$ the set of normalized $(\mu(x)=1) \phi$-invariant measures on $X$. Kryloff and Bogoliouboff introduced the notion of unique ergodicity to describe the situation in which $M_{\phi}$ reduces to a single point. We shall present here a generalization of this concept, illustrate its usefulness and discuss some examples.

1. Denote the measure entropy of $\phi$ with respect to $\mu \in M_{\phi}$ by $h_{\mu}(\phi)$ and set

$$
\hbar(\phi)=\sup _{\mu \in M \phi} h_{\mu}(\phi) .
$$

Definition. If $\bar{h}(\phi)<+\infty$ and there exists a unique $\bar{\mu} \in M_{\phi}$ such that

$$
h_{\bar{\mu}}(\phi)=\bar{h}(\phi)
$$

then $(X, \phi)$ is said to be an intrinsically ergodic system (i.e.s.).

Clearly a uniquely ergodic system with finite entropy is an i.e.s.; that the converse is not true may be seen already from the example of the bilateral 2-shift which is obviously not uniquely ergodic but is an i.e.s. We shall sketch two proofs of this well-known fact, since the methods are useful for later generalizations.

1. (After Parry [9].) Let $\alpha$ denote the basic partition of $X_{-\infty}^{\infty}\{0,1\}$ into two sets, $A_{0}, A_{1}$ where $A_{i}$ consists of all sequences with an $i$ in the zeroth place. $\alpha$ is a generator for the shift with respect to any regular shift invariant measure $m$ (see [10] for the facts and notations of entropy theory used here) and one deduces from the fact that equality holds in

$$
H_{m}\left(\alpha \bigvee \phi^{-1} \alpha\right) \leqq H_{m}(\alpha)+H_{m}\left(\phi^{-1} \alpha\right)
$$

only if $\alpha$ and $\phi^{-1} \alpha$ are independent, that a maximizing $m$ must be such that the coordinate functions are independent. Then an elementary computation reveals that there is a unique measure maximizing the entropy.

2. (After [2].) One observes that the number of sets in $\alpha, \phi^{-1} \alpha$, $\cdots, \phi^{-n+1} \alpha$ is $2^{n}$ and hence

AMS 1969 subject classifications. Primary 2870; Secondary 6002.

Key words and phrases. Unique ergodicity, intrinsic ergodicity, entropy, generic points, normal sequences. 


$$
H\left(\alpha \vee \phi^{-1} \alpha \vee \cdots \vee \phi^{-n+1} \alpha\right) \leqq \log 2^{n}
$$

with equality if and only if the measures of all sets in $\alpha \bigvee \phi^{-1} \alpha \bigvee \ldots$ $\vee \phi^{-n+1} \alpha$ are equal. Since the standard measure for the 2 -shift has this property it is the unique shift invariant measure with entropy $\log 2$.

Now assuming that $\bar{h}<+\infty,(X, \phi)$ can fail to be an i.e.s. for one of two reasons. It may be that there are many measures $\mu$ such that $h_{\mu}(\phi)=\bar{h}(\phi)$. Consider for example two copies of the two shift $X_{1}$ and $X_{2}$ and set $X=X_{1} \cup X_{2}$. Then it is easy to see that $\bar{h}=\log 2$ while we can distribute part of the standard measure on $X_{1}$ and part on $X_{2}$.

The second possibility is that there is no measure $\mu$ such that $h_{\mu}(\phi)=\bar{h}(\phi)$. This possibility was observed by Gurevich [8]. In case $X$ is closed shift invariant subset of $X_{-\infty}^{\infty}\{1,2, \cdots k\}$, and $\phi$ is the shift restricted to $X$ then this second possibility cannot occur, i.e. one can show that there exists some measure with $h_{\mu}(\phi)=\bar{h}(\phi)$. Basically the reason for this is that there exists a finite partition of $X_{-\infty}^{\infty}\{1,2, \cdots k\}$ into clopen sets that is a generator for the shift with respect to every measure.

2. The first application is a simple proposition a version of which was first used in [1], that shows how the isomorphism problem simplifies for i.e.s. Recall that the measure preserving transformations $(X, \phi, \mu)$ and $\left(X^{\prime}, \phi^{\prime}, \mu^{\prime}\right)$ are isomorphic if there exists an invertible measurable mapping $\nu$ from $X$ to $X^{\prime}$ so that $\phi^{\prime} \nu=\nu \phi$, and $\mu\left(\nu^{-1} A\right)=\mu^{\prime}(A)$ for all measurable subsets of $X$.

Proposition 1. Let $(X, \phi),\left(X^{\prime}, \phi^{\prime}\right)$ be a pair of i.e.s. such that $\bar{h}(\phi)=\bar{h}\left(\phi^{\prime}\right)$. Then if $\nu$ is an invertible measure transformation from $X$ to $X^{\prime}$ such that $\phi^{\prime} \nu=\nu \phi,(X, \phi, \mu)$ is isomorphic to $\left(X^{\prime}, \phi^{\prime}, \mu^{\prime}\right)$ where $\mu$ and $\mu^{\prime}$ are the measures of maximal entropy.

For the second illustration suppose that $(X, \phi)$ is an i.e.s. and $\nu: X \rightarrow X^{\prime}$ is equivariant with $\phi^{\prime}: X^{\prime} \rightarrow X^{\prime}$, i.e. $\phi^{\prime} \nu=\nu \phi$ where $\nu, \phi^{\prime}$ are continuous and $X^{\prime}$ is compact. Recall that $x \in X$ is said to be a generic point for a $\phi$-invariant measure $\mu$ if

$$
\frac{1}{n} \sum_{1}^{n} f\left(\phi^{k} x\right) \rightarrow \int f(x) \mu(d x)
$$

for all continuous functions $f$. Then we have

Proposition 2. In the situation described above if $x^{\prime}$ is a generic point for $\mu^{\prime}$ on $X^{\prime}$ with $h_{\mu^{\prime}}\left(\phi^{\prime}\right)=\bar{h}(\phi)$ then any $x \in \nu^{-1}\left(x^{\prime}\right)$ is a generic point for $\mu$. 
As an application of this last proposition one can obtain the results of V. N. Agafonov [3] on normal sequences, to wit that if a subsequence $\left\{n_{k}\right\}$ is defined by means of a finite automaton acting on the initial segments of a normal sequence $\xi_{1} \xi_{2}$. . (i.e. $n$ belongs to $\left\{n_{k}\right\}$ if and only if $\xi_{1} \xi_{2} \cdots \xi_{n}$ is accepted by the automaton) then $\xi_{n_{1+1}} \xi_{n_{2+1}+1} \xi_{n_{8}+1} \ldots$ is again a normal sequence. For the connection between generic points and results of this type, as well as a different approach see [5, Chapter 8]. Details of this application will appear elsewhere.

3. We discuss now some examples of i.e.s.

(1) Subshifts of finite type: (a) Let $X=X_{-\infty}^{\infty}\{1,2, \cdots k\}$ be endowed with the product topology, $\sigma: X \rightarrow X$ the shift, and $X_{A} \subset X$ a closed subset of $X$ defined by $A \subset \times_{1}^{m}\{1,2, \cdots k\}$ as follows:

$$
X_{\Lambda}=\{x \in X \text { :no } a \in A \text { occurs as an } m \text {-block in } X\} \text {. }
$$

Parry [9] showed that if $A$ is "aperiodic" then $\left(X_{A}, \sigma\right)$ is an i.e.s.

(b) A generalization of (a) has been suggested by H. Furstenberg. Let $G$ be a finite semigroup with generators $g_{1}, \cdots, g_{k}$ and a twosided zero $(0 \cdot g=g \cdot 0=0$ all $g \in G)$. An element $x \in X$ is $G$ admissible if for all $i \leqq j$

$$
g_{\xi_{i}} g_{\xi_{i+1}} \cdots g_{\xi_{j}} \neq 0
$$

where $x=\left\{\cdots \xi_{-1}, \xi_{0}, \xi_{1}, \cdots\right\}$. If $X_{G}$ is the set of $G$-admissible elements of $X$, then $X_{G}$ is shift invariant and if $G$ satisfies a certain "mixing" condition, that corresponds to the "aperiodicity" in the case (a) then $\left(X_{G}, \sigma\right)$ is an i.e.s. The proof follows the lines of the second proof in $\$ 1$.

(2) Ergodic automorphisms of compact groups are i.e.s. This was shown for the torus in [1] and in the general case by K. Berg [4]. A key fact in Berg's proof is that ergodic automorphisms are Kolmogorov automorphisms, and it is probable that those affine transformations on nil manifolds that are Kolmogorov automorphisms are i.e.s.

(3) Let $E=\left\{\left\{e_{i}^{(1)}\right\}, \cdots\left\{e_{i}^{(k)}\right\}\right\}$ be $k$ sequences of zeros and ones, we shall say that $x \in X_{-\infty}^{\infty}\{0,1, \cdots, k\}$ is $E$-admissible if

(i) the symbols $\{1, \cdots, k\}$ are isolated in $x$, i.e. they are preceded and followed by a zero.

(ii) If $\xi_{n}, \xi_{n+m} \in\{1,2, \cdots, k\}$ while $\xi_{n+i}=0, i=1, \cdots, m-1$, then $e_{m}^{\xi_{n}}=1$, where $x=\left(\cdots \xi_{-1}, \xi_{0}, \xi_{1}, \cdots\right)$. The shift restricted to the closure of the set of $E$-admissible sequences in an i.e.s. (if the $\left\{e_{i}^{(j)}\right\}$ are "aperiodic") and $\bar{h}=\log c$ where $c$ is the unique positive root of 


$$
\sum_{1}^{\infty}\left(\sum_{j=1}^{k} e_{i}^{(j)}\right) t^{-i}=t .
$$

The proof of this fact follows the lines of the first proof of the i.e.s. of the 2-shift. Thus we can obtain easily an i.e.s. with arbitrary values for $\bar{h}$, a fact not readily evident from examples (1) and (2).

Dinaburg [5] has made use of these sets (for $k=1$ ) in calculating the topological entropy of certain $C^{\infty}$-diffeomorphisms restricted to a part of their nonwandering set.

In all of the examples known up to now $\bar{h}(\phi)$ equals the topological entropy of $\phi$. Goodwyn [7] has proved that $\bar{h}(\phi) \leqq h_{\text {top }}(\phi)$, while the example in [8] shows that there need not be any measure $\mu$ in $M_{\phi}$ with

$$
h_{\mu}(\phi)=h_{\text {top }}(\phi) \text {. }
$$

Nonetheless, there is some evidence for the following conjecture with which we conclude:

Conjecture. If $X$ is a closed invariant subset of a finite shift $\sigma$, such that $M_{\sigma}$ contains a measure $\mu$ satisfying

(1) $(X, \mu, \sigma)$ is a $K$-automorphism;

(2) $\mu$ assigns positive measure to open sets of $X$, then $(X, \sigma)$ is an i.e.s. and $\bar{h}$ equals the topological entropy of $(X, \sigma)$.

\section{REFERENCES}

1. R. L. Adler and B. Weiss, Entropy, a complete metric invariant for automorphisms of the torus, Proc. Nat. Acad. Sci. U.S.A. 57 (1967), 1573-1576, MR 35 \#3031.

2. - Similarity of automorphisms of the torus, Mem. Amer. Math. Soc. No. 98 (1970).

3. V. N. Agafonov, Normal sequences and finite automata, Dokl. Akad. Nauk SSSR 179 (1968), 255-256 = Soviet Math. Dokl. 9 (1968), 324-325. MR 38 \#6893.

4. K. R. Berg, Convolution of invariant measures. Maximal entropy, Math. Systems Theory 3 (1969), 146-150.

5. E. I. Dinaburg, An example of the computation of topological entropy, Uspehi Mat. Nauk 23 (1968), no. 4 (142), 249-250. (Russian) MR 37 \#5883.

6. H. Furstenberg, Stationary processes and prediction theory, Ann. of Math. Studies, no. 44, Princeton Univ. Press, Princeton, N.J., 1960. MR 25 \#3573.

7. L. W. Goodwyn, Topological entropy bounds measure-theoretic entropy, Proc. Amer. Math. Soc. 23 (1969), 679-688.

8. B. M. Gurevič, Topological entropy of enumerable Markov chains, Dokl. Akad. Nauk SSSR 187 (1969), 715-618=Soviet Math, Dokl. 10 (1969), 911-915.

9. W. Parry, Intrinsic Markov chains, Trans. Amer. Math. Soc. 112 (1964), 55-66. MR 28 \#4579.

10. V. A. Rohlin, Lectures on the entropy theory of transformations with invariant measure, Uspehi Mat. Nauk 22 (1967), no. 5 (137), 3-56=Russian Math. Surveys 22 (1967), no. 5, 1-52. MR 26 \#349.

Hebrew University, Jerusalem, Israfel 